\title{
Dermatosis perforante adquirida, presentación de casos en pacientes con neoplasias malignas
}

\section{Acquired perforating dermatosis, a report of cases in patients with malignant neoplasms}

\author{
M. Noelia Andrade*, M. Florencia Alfaro, Leandro Van Caester, Valeria E. Rossello, Verónica Gallerano, \\ Mónica Herrero y Susana B. Pereyra
}

Servicio de Dermatología del Hospital Córdoba, Córdoba, Argentina

\section{Resumen}

Se presentan dos casos diagnosticados de dermatosis perforante adquirida (DPA) en el contexto de pacientes con neoplasias malignas, en los que se confirmó la resolución de la clínica cutánea luego de la mejoría oncológica. Se identificaron pocos informes de casos que relacionaran la DPA con ampulomas o linfomas de Hodgkin, como estos pacientes; se plantea que es posible esta relación como anomalía paraneoplásica, pero se necesitan estudios adicionales para confirmarlo.

Palabras clave: Dermatosis perforante adquirida. Ampuloma. Linfoma de Hodgkin. Paraneoplasia.

\begin{abstract}
We present two cases of acquired perforating dermatosis (DPA), in patients with associated malignancies, where resolution of cutaneous symptoms was confirmed after oncological improvement. We found few reports of cases worldwide relating DPA with ampuloma and Hodgkin's lymphoma as our patients; It is suggested that this association may occur as a paraneoplastic condition, but additional studies are necessary to confirmation.
\end{abstract}

Key words: Acquired perforating dermatosis. Ampuloma. Hodgkin's lymphoma. Paraneoplastic.

\section{Introducción}

La dermatosis perforante adquirida (DPA) es un trastorno cutáneo que a menudo se vincula con enfermedades sistémicas subyacentes, como diabetes mellitus, insuficiencia renal crónica o proceso maligno. Se presentan dos casos acompañados de neoplasias malignas, uno de ellos con ampuloma y otro con linfoma de Hodgkin (LH), que luego de su tratamiento experimentaron resolución de la DPA.

\section{Casos clínicos}

El primer paciente, un varón de 63 años de edad con antecedentes de diabetes tipo 2, hipertensión arterial y obesidad, se interna en la sala común por colestasis, insuficiencia renal aguda, anemia y descompensación diabética. Durante la internación se diagnosticó un adenocarcinoma de ampolla duodenal (ampuloma) moderadamente diferenciado invasor. En virtud del prurito cutáneo intenso se interconsultó con el servicio de 
dermatología, cuya exploración física cutánea reveló múltiples pápulas eritematosas con tapón hiperqueratósico central, generalizadas y con predominio en tronco y extremidades (Fig. 1).

Los estudios de laboratorio al ingreso de la hospitalización mostraron alteraciones en la creatinina (2.26 mg/dl) que mejoraron durante la internación, glucemia $(210 \mathrm{mg} / \mathrm{dl})$ que mantuvo valores elevados con difícil control diabético, hemoglobina (8.1\%), bilirrubina total $(2.47 \mathrm{mg} / \mathrm{dl})$, bilirrubina directa $(2.19 \mathrm{mg} / \mathrm{dl})$, fosfatasa alcalina $(652 \mathrm{mg} / \mathrm{dl})$, gammaglutamil transpeptidasa (150 mg/dl), CA 19-9 (282.5 U/L), que mejoraron de forma paulatina con el tratamiento oncológico; el antígeno carcinoembrionario y la fetoproteína $\alpha$ fueron normales.

La segunda paciente, una mujer de 20 años de edad con antecedente patológico de $\mathrm{LH}$, variedad esclerosis nodular (estadio IIIb), resistente a tres esquemas quimioterápicos con indicación de brentuximab, se encontraba a la espera de trasplante autólogo de médula ósea. Consultó por dermopatía muy pruriginosa de un mes de evolución. La exploración física presentó lesiones cutáneas de iguales características que el primer paciente, pero con localización predominante en las piernas (Fig. 2). Ningún paciente tuvo compromiso de rostro, palmas, plantas y mucosas. En los estudios de laboratorio de la segunda paciente se reconoció anemia de los procesos crónicos con hemoglobina de $10.8 \mathrm{mg} / \mathrm{dl}$ y lactato deshidrogenasa de $1,770 \mathrm{U} / \mathrm{L}$, que mejoraron luego de la compensación oncológica.

Se realizó biopsia cutánea en ambos pacientes y se observó en el estudio histopatológico con tinción de hematoxilina eosina hiperplasia epidérmica con depresión cupuliforme que contenía queratina, detritos celulares entremezclado con infiltrado inflamatorio, así como eliminación transepidérmica de fibras de colágeno (Fig. 3). La tinción de tricrómico de Masson mostró fibras de colágeno que penetran a través de la epidermis intacta (Fig. 4). En ningún caso se encontró eliminación de fibras elásticas con tinción de orceína.

Se indicó en ambos casos crema con clobetasol al $0.05 \%$ tópica dos veces por día, e hidroxicina $(50 \mathrm{mg}$ cada 12 h) con mejoría clínica parcial. En el primer caso se practicó una duodenopancreatectomía y el segundo comenzó con brentuximab oral. Ambos pacientes mostraron alivio del prurito y la dermopatía con el tratamiento y recuperación de la enfermedad oncológica, con resolución en aproximadamente un mes. Los dos casos evolucionaron con cicatrices hiperpigmentadas residuales.

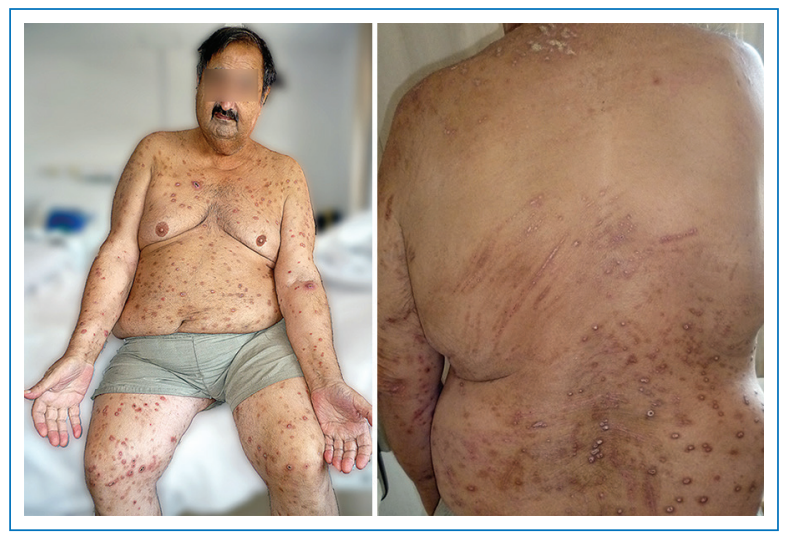

Figura 1. Múltiples pápulas eritematosas generalizadas con tapón hiperqueratósico central. En el dorso medio se observa el fenómeno de Koebner (caso 1).

\section{Discusión}

Las dermatosis perforantes o enfermedades por eliminación transepitelial son un grupo de enfermedades heterogéneas caracterizadas por la extrusión transepidérmica de material dérmico. Pueden ser hereditarias, cuya presentación es rara y aparecen en niños de manera recurrente con resolución espontánea. Las formas adquiridas surgen en la adultez con tendencia a relacionarse con enfermedades sistémicas y es el patrón más frecuente $e^{1-4}$. Con anterioridad se clasificaban según fueran el material eliminado (colágeno, tejido elástico o tejido conectivo necrótico) y el tipo de perforación (transepidérmica o transfolicular) en cuatro entidades: elastosis perforante serpiginosa, foliculitis perforante, enfermedad de Kyrle y colagenosis perforante reactiva ${ }^{5,6}$. Debido a que las características clínicas e histológicas de las DPA no son uniformes y pueden parecerse a cualquiera de los cuatro trastornos perforantes típicos, en la actualidad muchos autores prefieren el uso del término DPA para referirse a las dermatosis perforantes del adulto, en el contexto de un paciente sin antecedentes familiares de dermatosis perforante y con enfermedad sistémica adjunta. Se prefieren los términos colagenosis perforante reactiva y elastoma perforante serpiginoso para las formas hereditarias $^{6,7}$. Tienen distribución mundial y su incidencia es similar en ambos sexos y edades, con un punto máximo en la adolescencia ${ }^{5,8}$.

La etiopatogenia de DPA se desconoce, pero se calcula que el traumatismo superficial, la microangiopatía dérmica y la acumulación de sustancias que no pueden eliminarse por diálisis son quizá los factores desencadenantes principales en individuos predispuestos ${ }^{1,5,6,9}$. 


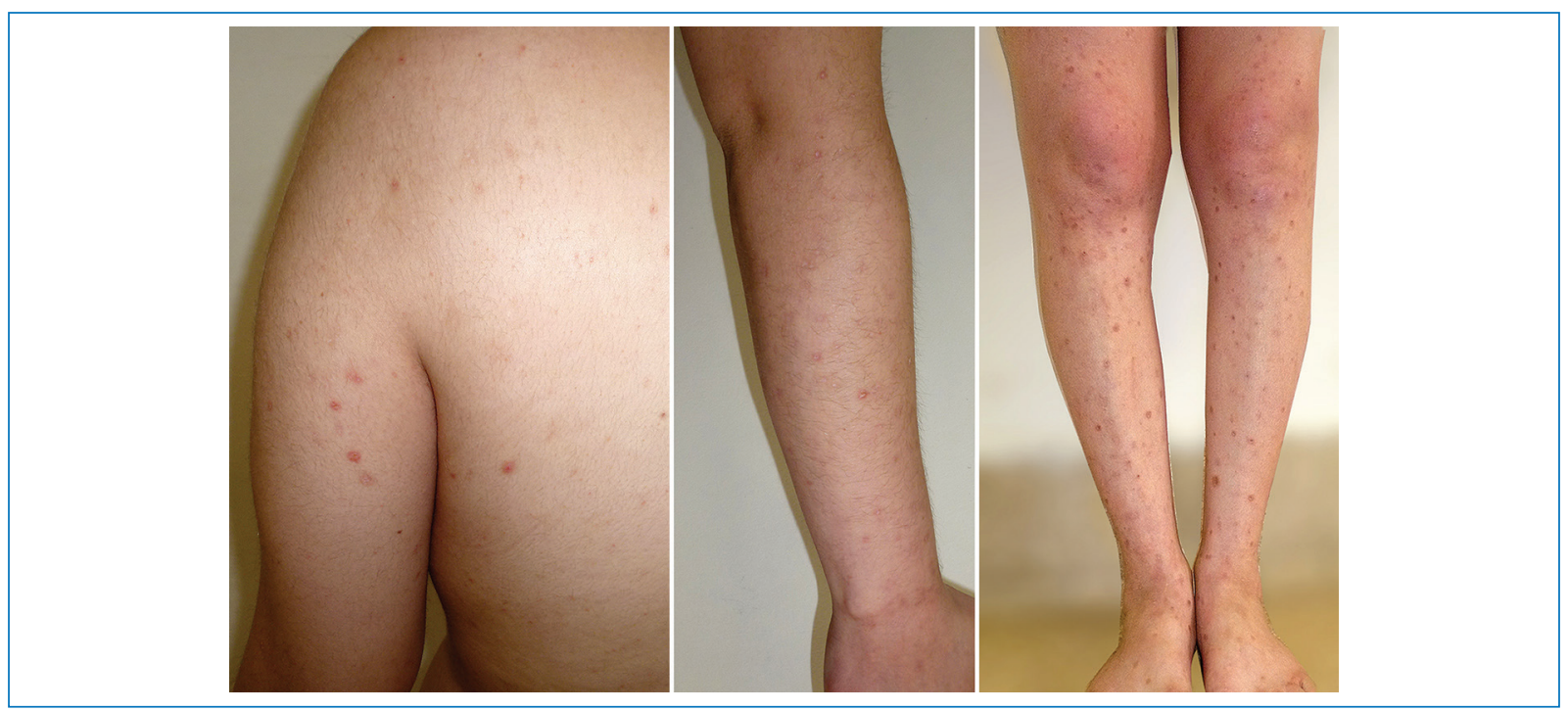

Figura 2. Pápulas eritematosas con tapón hiperqueratósico central, que comprometen dorso y miembros superiores, con predominio en miembros inferiores (caso 2).

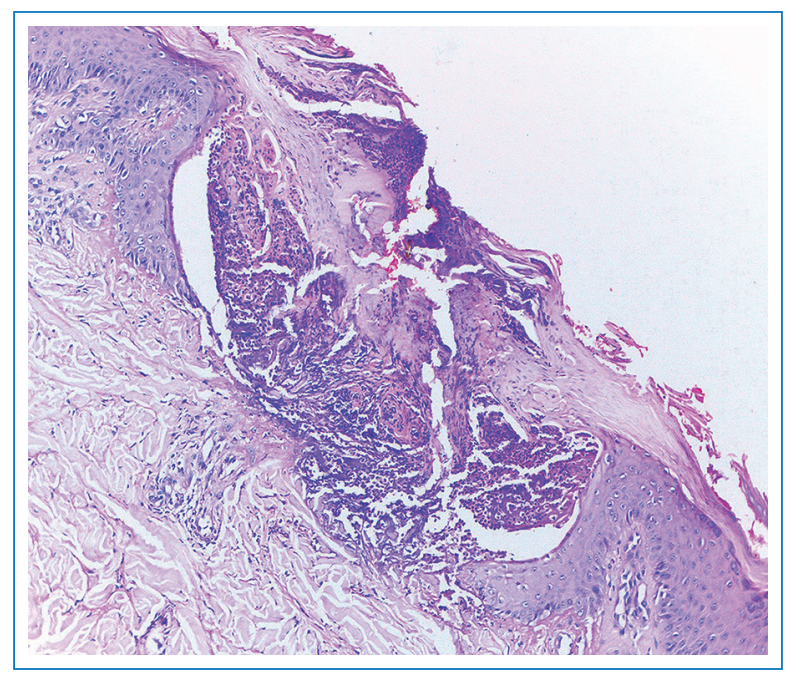

Figura 3. Se observa hiperqueratosis epidérmica y una invaginación llena de restos celulares, queratina, fibras de colágeno basófilas y leucocitos polimorfonucleares neutrófilos (tinción de hematoxilina-eosina).

La fibronectina, que tiene un papel en la señalización, transporte y diferenciación de las células epiteliales, puede estar aumentada en la DPA y causar proliferación y perforación del epitelio ${ }^{1,8}$. Asimismo, se ha referido en la fisiopatogenia el metabolismo anormal de las vitaminas $A \circ D^{5,8}$, liberación de enzimas de neutrófilos y expresión anormal de metaloproteinasas y factor de crecimiento tranformante- $\beta 3^{7-9}$. La DPA puede desarrollarse ante traumatismos menores como el rascado ${ }^{1-3,5,10}$,

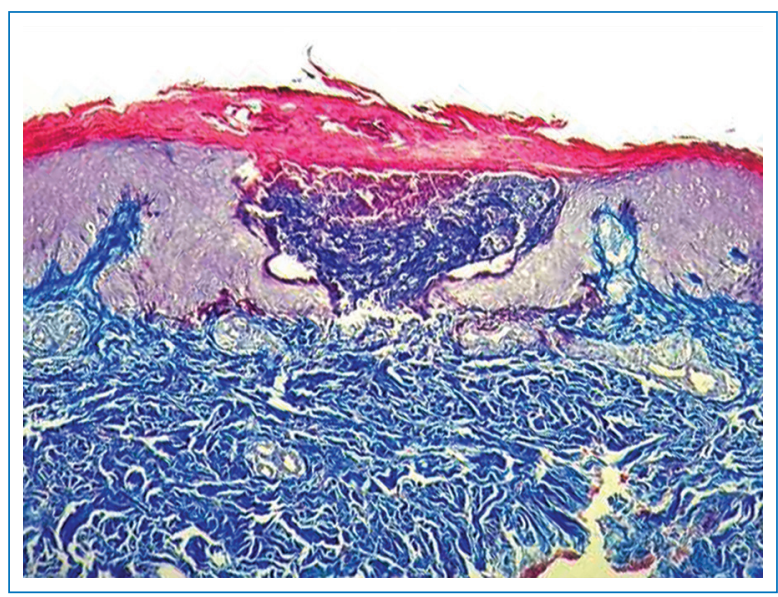

Figura 4. Se observan fibras de colágeno que penetran a través de la epidermis (tinción de tricrómico de Masson).

mordeduras de artrópodos y escabiosis ${ }^{3,5}$. En mayor medida se relaciona con enfermedades sistémicas, en particular con diabetes mellitus e insuficiencia renal crónica ${ }^{1,5,6,10,11}$, sobre todo en pacientes en hemodiálisis crónica $^{3,5,6}$. Relaciones menos frecuentes son la insuficiencia hepática, la dermatitis atópica, las endocrinopatías como el hipotiroidismo y el hiperparatiroidismo, las enfermedades infectocontagiosas como el virus de la inmunodeficiencia humana y la tuberculosis, las autoinmunitarias como el lupus eritematoso sistémico, la vasculitis y la dermatomiositis, y las neoplasias ${ }^{1,3-6,10,12-14}$. 
Respecto de esta última, se han descrito casos en las publicaciones médicas de DPA relacionada con carcinoma de tiroides, mama, pulmón, hígado y vías biliares ${ }^{1,3,8,12-14}$, además de ampuloma ${ }^{12}$ y LH $^{2,10,15}$, como presentaron estos pacientes. Se plantea la hipótesis de que la DPA es una anomalía secundaria a la enfermedad linfoproliferativa o biliar debido al traumatismo del rascado en respuesta al prurito $2,3,9,10,11,15,16$. Sin embargo, aún no se ha dilucidado si las alteraciones metabólicas de estas enfermedades se traducen en un cambio patológico a la DPA, si el prurito es predisponente y hasta qué punto los episodios inmunológicos o bioquímicos precipitan la enfermedad ${ }^{9,12}$. Además, se ha conjeturado que puede ocurrir una DPA como afección paraneoplásica; algunos autores han recomendado considerar una tumoración en pacientes con esta anomalía, por lo que se necesitan estudios adicionales para conocer la relación $n^{3,12}$.

En clínica se manifiesta con múltiples pápulas queratósicas umbilicadas eritematosas ${ }^{1,10}$ muy pruriginosas y es posible identificar el fenómeno de Koebner por rascado. Se resuelven habitualmente con hiperpigmentación ${ }^{4-6,14}$. Se localizan sobre todo en tronco y extremidades, en particular en las superficies extensoras y algunas veces en cuello y rostro $0^{1,3-6,10}$.

En las etapas iniciales, la histopatología muestra leve acantosis de la epidermis y ensanchamiento de la dermis papilar con haces de colágeno degenerados con basofilia. A medida que la enfermedad evoluciona, se forman típicas invaginaciones cupuliformes de la epidermis orientadas de modo perpendicular hacia la superficie, llenas de células paraqueratósicas, queratina, fibras de colágeno degeneradas basófilas y detritos celulares. Puede haber un ligero infiltrado inflamatorio perivascular mononuclear en la dermis $^{1,2,5,9,10}$.

Los diagnósticos diferenciales se establecen con prurigo nodular ${ }^{1,3}$, liquen plano hipertrófico, queratoacantomas eruptivos, enfermedad de Darier, queratosis pilar y otras dermatosis perforantes de materiales exógenos o endógenos. La histopatología debe confirmar el diagnóstico clínico ${ }^{3,8}$.

El tratamiento de este grupo de afecciones está dirigido a disminuir el prurito y mejorar las condiciones de la enfermedad subyacente; empero, debido a que los mecanismos patogénicos que intervienen en su aparición no se han aclarado por completo, aún es empírico, difícil y en ocasiones poco satisfactorio ${ }^{1,9}$. Se ha demostrado que el tratamiento combinado, en lugar de la monoterapia, produce resultados más favorables. Los emolientes como crema de urea, corticosteroides de baja y mediana potencia, queratolíticos como pomadas con tretinoína y preparaciones con salicilato, y antihistamínicos $\mathrm{H} 1$ orales son útiles para aliviar la picazón. En caso de respuesta discreta, debe considerarse el tratamiento sistémico: retinoides, corticoides, talidomida, doxiciclina, rifampicina, amitriptilina, alopurinol y fototerapia (en especial ultravioleta $B$ de banda estrecha y ultravioleta $A$ con soraleno), según sean la enfermedad primaria y el estado general del paciente ${ }^{1,4,8}$.

\section{Conclusiones}

Las dermatosis perforantes son un grupo de enfermedades heterogéneas que muestran eliminación transepidérmica de material dérmico y se relacionan tanto con traumatismos locales como con enfermedades sistémicas, entre ellas las malignas oncológicas. En los casos presentados, la DPA se resolvió con la mejoría de la enfermedad oncológica subyacente, lo que sustenta la teoría de otros autores que informaron casos similares, según la cual dicho episodio podría representar un verdadero fenómeno paraneoplásico. Existen varias teorías acerca de su mecanismo etiopatiogénico, pero se requieren más estudios para catalogarla como paraneoplásica.

\section{Financiamiento}

Los autores no recibieron financiamiento alguno para la realización del manuscrito.

\section{Conflicto de intereses}

Los autores no presentan ningún conflicto de intereses.

\section{Responsabilidades éticas}

Protección de personas y animales. Los autores declaran que para esta investigación no se han realizado experimentos en seres humanos ni en animales.

Confidencialidad de los datos. Los autores declaran que han seguido los protocolos de su centro de trabajo sobre la publicación de datos de pacientes.

Derecho a la privacidad y consentimiento informado. Los autores han obtenido el consentimiento informado de los pacientes y/o sujetos referidos en el 


\section{artículo. Este documento obra en poder del autor de correspondencia.}

\section{Bibliografía}

1. Karpouzis A, Giatromanolak A, Sivridis E, Kouskoukis C. Acquired reactive perforating collagenosis: current status. Journal of Dermatology. 2010;37:585-92.

2. Pedragosa R, Knobel HJ, Huquet $P$, Oristrell J, Valdés M, Bosch JA. Reactive perforating collagenosis in Hodgkin's disease. The Amenrican Jornal of Dermatopathology. 1987;9(1):41-4410.

3. Yazdi S, Saadat P, Young S, Hamidi R, Vadmal MS. Acquired reactive perforating collagenosis associated with papillary thyroid carcinoma: a paraneoplastic phenomenon? Clin Exp Dermatol. 2010;35(2):152-5.

4. González-Lara L, Gómez-Bernal S, Vázquez-López F, Vivanco-Allende B. Dermatosis perforante adquirida: presentación de 8 casos. Actas Dermosifiliogr. 2014;105(6):39-43.

5. Sehgal VN, Jain S, Thappa DM, Bhattacharya SN, Logani K. Perforating dermatoses: a review and report of four cases. J Dermatol. 1993.20(6):329-40.

6. Saray Y, Seçkin D, Bilezikçi B. Acquired perforating dermatosis: clinicopathological features in twenty-two cases. J Eur Acad Dermatol Venereol. 2006 Jul;20(6):679-88.

7. Arnaudin D, Matamoros E, Campoy MV, Díaz Leaño C, Michelena M, Arena $G$, et al. Dermatosis perforante adquirida, presentación de un caso. Arch Argent Dermatol. 2014;64(6):239-43
8. Shahidi-Dadras M, Hashemi R, Saeidpour M, Khademi F. Acquired reactive perforating collagenosis in association with pulmonary papillary adenocarcinoma. Iran J Dermatol. 2013;16:69-72.

9. Gambichler T, Birkner L, Stucker M, Othlinghaus N, Itmeyer P, Kreuter A Up-regulation of transforming growth factor-b3 and extracellular matrix proteins in acquired reactive perforating collagenosis. J Am Acad Dermatol 2009:60:463-9.

10. Rivera-Rodríguez A, Prieto-Torres L, Felipo-Berlanga F, Ara-Mart M. Acquired reactive perforating collagenosis associated with Hodgkin disease. Clinical and Experimental Dermatology. 2017:42:934-6.

11. Satti MB, Aref AH, Raddadi AA, Al-Ghamdi FA. Acquired reactive perforating collagenosis: a clinicopathologic study of 15 cases from Saudi Arabia. J Eur Acad Dermatol Venereol. 2010;24(2):223-7.

12. Chae KS, Park YM, Cho SH, Cho BK. Br. Reactive perforating collagenosis associated with periampullary carcinoma. $J$ Dermatol 1998:139(3):548-50.

13. Kim RH, Kwa M, Adams S, Meehan SA, Stein JA. Giant acquired reactive perforating collagenosis in a patient with diabetes mellitus and metastatic breast carcinoma. JAAD Case Rep. 2016;2(1):22-4.

14. Lukács J, Schliemann S, Elsner P. Treatment of acquired reactive perforating dermatosis - a systematic review. J Dtsch Dermatol Ges. 2018; 16(7):825-42.

15. Eigentler TK, Metzler G, Brossart P, Fierlbeck G. Acquired perforating collagenosis in Hodgkin's disease. J Am Acad Dermatol. 2005;52:922.

16. Lee YS, Vijayasingam S, Tan YO, Wong ST. Acquired perforating dermatosis associated with recurrent hepatocellular carcinoma. Int J Dermatol. $1996 ; 35(10): 743-5$ 